\title{
Article
}

\section{Soft X-ray Lensless Imaging in Reflection Mode}

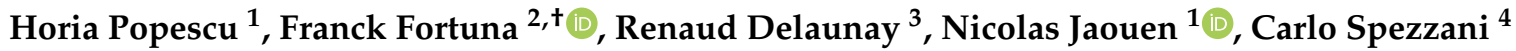 \\ and Maurizio Sacchi ${ }^{1,5, * \text { (D) }}$
}

1 Synchrotron SOLEIL, Saint-Aubin, B.P. 48, 91192 Gif-sur-Yvette, France; horia.popescu@synchrotron-soleil.fr (H.P.); nicolas.jaouen@synchrotron-soleil.fr (N.J.)

2 Centre de Sciences Nucléaires et Sciences de la Matière, CNRS-UMR 8609, Université Paris-Sud, Bât. 104, 91405 Orsay, France; franck.fortuna@u-psud.fr

3 Laboratoire de Chimie Physique-Matière et Rayonnement, CNRS-UMR 7614, Sorbonne Université, 75005 Paris, France; renaud.delaunay@sorbonne-universite.fr

4 ELETTRA, Sincrotrone Trieste, Area Science Park, 34149 Basovizza, Trieste, Italy; carlo.spezzani@elettra.eu

5 Institut des Nanosciences de Paris, CNRS-UMR 7588, Sorbonne Université, 75005 Paris, France

* Correspondence: maurizio.sacchi@synchrotron-soleil.fr

† Present address: Institut des Sciences Moléculaires d'Orsay, CNRS-UMR 8214, Université Paris-Saclay, Bât. 520, 91405 Orsay, France.

\section{check for} updates

Citation: Popescu, H.; Fortuna, F.; Delaunay, R.; Jaouen, N.; Spezzani, C.; Sacchi, M. Soft X-ray Lensless Imaging in Reflection Mode. Photonics 2021, 8, 569. https://doi.org/ $10.3390 /$ photonics 8120569

Received: 16 November 2021 Accepted: 9 December 2021 Published: 11 December 2021

Publisher's Note: MDPI stays neutral with regard to jurisdictional claims in published maps and institutional affiliations.

Copyright: (c) 2021 by the authors. Licensee MDPI, Basel, Switzerland. This article is an open access article distributed under the terms and conditions of the Creative Commons Attribution (CC BY) license (https:/ / creativecommons.org/licenses/by/ $4.0 /)$.

\begin{abstract}
We report on the development and implementation of methodologies dedicated to soft X-ray imaging by coherent scattering in reflection mode. Two complementary approaches are tested, based on Fourier transform holography and on ptychography. A new method for designing holographic masks has been developed. Our results represent a feasibility test and highlight the potential and limitations of imaging in reflection mode. Reflectivity is less efficient than transmission at soft X-ray wavelengths, hampering the acquisition of good quality images. Nonetheless, it has the potential to image a wider set of samples, notably those that are not transparent to soft X-rays. Although the images obtained so far are of modest quality, these results are extremely encouraging for continuing the development of coherent soft X-ray imaging in reflection mode.
\end{abstract}

Keywords: soft X-ray imaging; holography; ptychography; reflection geometry

\section{Introduction}

Interest in developing instrumentation and methods for soft $\mathrm{X}$-ray imaging via coherent scattering is supported by a well-documented activity of modern X-ray sources, namely synchrotron, free-electron laser (FEL) and high-harmonic generation (HHG) facilities. In general, techniques relying on the elastic X-ray scattering, like resonant reflectivity, diffuse scattering, diffraction and lensless microscopy, which provide a rather unique tool-set for the investigation of timely and important topics relating to the ordering and to the dynamics of spin, charge and orbital moment in complex materials.

Tunable soft $\mathrm{X}$-ray sources make it possible to select photon energies that match core resonances, merging the structural sensitivity of scattering with the element selectivity and sensitivity to electronic properties of a spectroscopic tool [1]. The soft $X$-ray range covers the relevant resonances of all the elements important for magnetic materials, notably the $2 p \rightarrow 3 d$ of $3 d$ transition metals (TM) and the $3 d \rightarrow 4 f$ of rare-earths (RE). The polarization tunability introduces a well-defined and selectable symmetry on the X-ray probe that can be used to reveal and study specific symmetries of the sample. Resonant magnetic scattering of polarized $X$ rays (XRMS) is a rather unique tool for studying both ferromagnetic (FM) and antiferromagnetic (AF) order, offering a view of the magnetic structure that is paralleled only by polarized neutron reflectivity. Resonant scattering in reflection mode (measuring the specular, diffuse or diffracted intensity) addresses the electronic properties, the microstructure [2,3], the magnetic structure [4-10], as well as the charge [11-13] and orbital $[3,14]$ order with in-depth resolution $[15,16]$. 
Sources characterized by large transverse coherence can be used to produce X-ray images with high spatial resolution via scattering measurements. Over the last decade, several of the approaches to coherent imaging that were developed in the visible range have been extended successfully to the X-ray range, notably Fourier transform holography (FTH) $[17,18]$ and ptychography [19-21]. Central to interest in imaging via coherent soft $X$-ray scattering is the study of magnetic structures (and of their dynamics), a topic of high impact in both fundamental research and applications [22].

Imaging by soft $X$-ray scattering takes full advantage of all the peculiar characteristics of modern X-ray sources, especially in terms of coherence and brilliance. Also, flux is a critical parameter, since low intensity can be a limiting factor for new developments in imaging techniques; as will become apparent in the following, this is particularly true in reflection mode.

From the very beginning [17] and up to now, soft X-ray FTH experiments have been performed intensely in transmission mode, and several complementary technical solutions have been developed: integrated $[17,23]$ or separate $[18,24]$ sample/mask approach, outof-plane [24] or in-plane [25,26] magnetization analysis, use of circular (large majority of published works) or linear [27] polarization of the $\mathrm{X}$ rays. Many studies took advantage also of the optimized experimental conditions at FEL sources for two-color [28] or singleshot $[29,30]$ magnetic imaging.

The transmission mode, however, suffers from one main limitation, i.e., the sample must be transparent. In the soft $X$-ray range this means a total thickness (i.e., including sample and substrate) of at most a few hundreds of $\mathrm{nm}$, which hampers or even impedes the study of a large class of samples, notably single crystals and epitaxial thin films. The implementation of X-ray imaging in reflection mode has the potential to overcome constraints on the sample transparency, extending these imaging techniques to a wider class of materials.

Microscopy in reflection mode at extreme ultraviolet wavelengths has been performed by coherent diffraction imaging, relying on iterative phase retrieval algorithms. Examples were reported, for instance, at HHG sources, using $29 \mathrm{~nm}$ [31] and $42.6 \mathrm{~nm}$ [32] radiation.

Performing soft $\mathrm{X}$-ray holography in reflection mode has been attempted at several synchrotron sources over the last 10 years, with scarce success. The simple idea of replacing the reference aperture (pin-hole) by a reference pin is hindered by the overwhelming contribution of diffuse scattering from the substrate. No significant result has been reported to date using this approach. The only published example of FTH in reflection used a holographic mask, placed after the sample surface and normal to it, that intercepts the scattered beam on its way to the detector [33]. This geometry limits severely the area that can be imaged to a fixed zone next to the sample edge. Moreover, since the mask is far from the X-ray impact spot, one has to rely on phase-retrieval algorithms for attaining an acceptable spatial resolution [34]. This first example of FTH in reflection [33] was a very important result of high impact, but the proposed configuration proved rather impractical and did not find many applications.

The objective of our work is to continue developing and testing new methods for reflection soft $\mathrm{X}$-ray imaging using holography and ptychography. The former requires a very simple data treatment, but it is extremely photon-hungry since it relies on the intensity of a reference beam generated by a tiny aperture, the size of which limits the final spatial resolution [17]. The latter is simpler to implement experimentally, but requires using phase retrieval algorithms for image reconstruction [35-37]. Given the technical similarities of the two approaches, it is easy to implement both in a single experimental setup.

\section{Materials and Methods}

We have developed a setup for X-ray imaging in reflection mode based on the separate mask/sample scheme, making it possible to bring any part of the sample into the field of view. We installed the new set-up in the IRMA-2 scattering chamber [38] and we tested it at the SEXTANTS beamline [39] of the SOLEIL synchrotron. The X-ray transverse 
coherence was defined by selecting the central part of the undulator emission cone via a four-jaws device, a configuration already characterized [38] and tested in previous experiments [26,27]. Additionally, the incoming beam was skimmed by placing a $20 \mu \mathrm{m}$ guard-hole $\sim 10 \mathrm{~mm}$ before the mask/sample setup in order to limit the irradiated mask area. The two-dimensional detector was a $27.6 \times 27.6 \mathrm{~mm}^{2}$ charge-coupled device (CCD) with $2048 \times 2048$ pixels. A $100 \mathrm{~nm}$-thick aluminum filter protected the detector from the stray visible light generated by the in-vacuum encoders. The CCD could be mounted on fixed flanges at either $30^{\circ}$ or $90^{\circ}$ from the incoming beam axis in order to collect the specular and the diffuse intensity from the sample set at either $\omega=15^{\circ}$ or $\omega=45^{\circ}$ incidence. In both cases, the detector was positioned at $470 \mathrm{~mm}$ from the sample, collecting $\pm 1.6^{\circ}$ along the two directions in the YZ' plane normal to the specular beam axis. Since the projection of the scattering vector $\mathbf{Q}$ on the sample surface depends on $\omega$ in the $Z^{\prime}$ direction but not in the $Y$ direction, a square detector covers different ranges of $Q_{Y}$ and $Q_{Z^{\prime}}$ in the reciprocal space, the difference increasing for lower $\omega$ values.

Test samples were arrays of sub-micron objects of different materials, namely an $80 \mathrm{~nm}$ thick $\mathrm{Fe}_{20} \mathrm{Ni}_{80}$ (permalloy, or Py) layer, a $50 \mathrm{~nm}$ thick FeRh layer, and a $\left(\mathrm{Co}_{11 \mathrm{~nm}} / \mathrm{Cu}_{20 \mathrm{~nm}}\right)_{20}$ multilayer. The patterns consisted of squares, of rectangles, and of triangular holes. The Py and $\mathrm{Co} / \mathrm{Cu}$ objects were prepared on $\mathrm{Si}$ substrates using X-ray lithography in combination with sputter deposition and lift-off according to the process described in [40]. The FeRh film was sputter-deposited on $\mathrm{MgO}$ and patterned using optical lithography and ion etching.

We used two different kinds of masks for holography and ptychography, both prepared by etching X-ray opaque supports $\left(1.2 \mu \mathrm{m}\right.$ of $\mathrm{AuCr}_{0.2}$ on $100 \mathrm{~nm}$ thick $\mathrm{Si}_{3} \mathrm{~N}_{4}$ membranes) using focused ion beam (FIB) milling. The final set-up for the synchrotron experiment is sketched in Figure 1: a $20 \mu \mathrm{m}$ pin-hole delimits the size of the beam reaching the mask and impinging on the sample, tilted at the desired angle $\left(45^{\circ}\right.$ in the picture for holography).
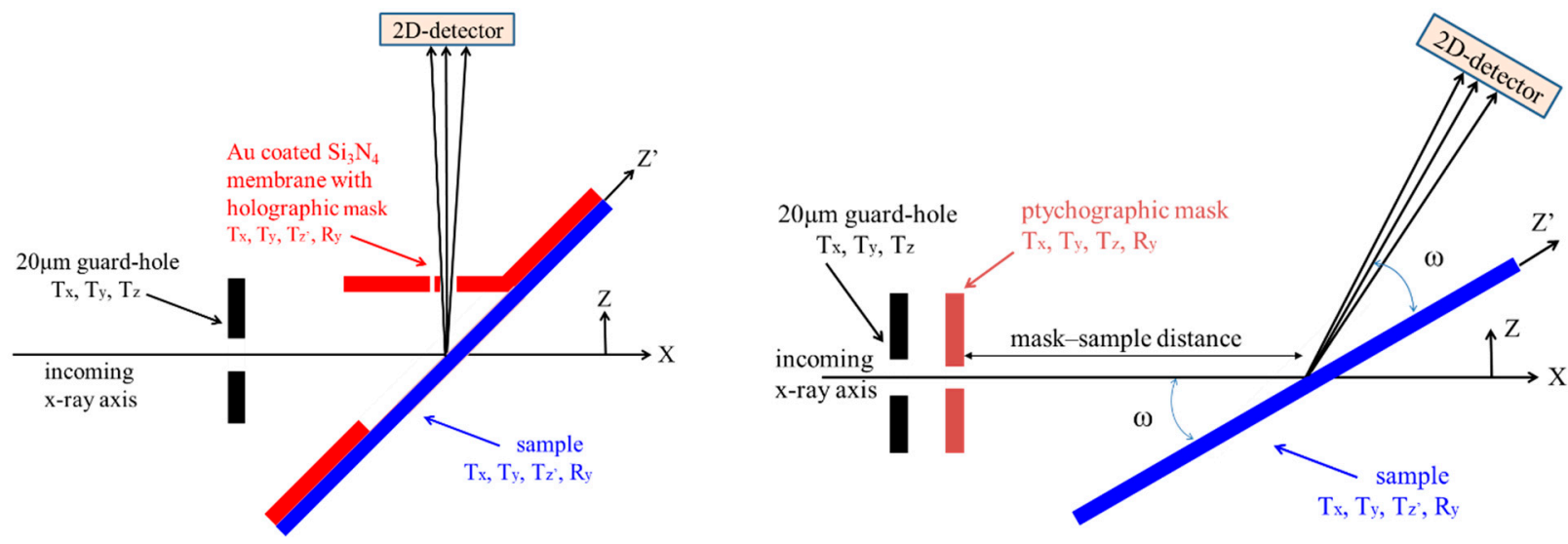

Figure 1. Sketch of the experimental set-up (side view) for holography (left) and ptychography (right), showing the three main elements (guard-hole, mask and sample) and their respective in-vacuum motorized translations (T) and rotations (R). The $\mathbf{Z}^{\prime}$ axis depends on $\omega$, the angle of grazing incidence $\left(\omega=45^{\circ}\right.$ for holography, $\omega=15^{\circ}$ and $45^{\circ}$ for ptychography). The $\mathbf{Y}$ axis, common to all elements, is perpendicular to the drawing.

An ensemble of 11 encoded translation and rotation tables, driven by either stepper or piezoelectric motors, provided all the required degrees of freedom to the guard-hole $\left(\mathrm{T}_{\mathrm{x}} \mathrm{T}_{\mathrm{y}} \mathrm{T}_{\mathrm{z}}\right)$, holographic or ptychographic mask $\left(\mathrm{T}_{\mathrm{x}} \mathrm{T}_{\mathrm{y}} \mathrm{T}_{\mathrm{z}} \mathrm{R}_{\mathrm{y}}\right)$ and sample $\left(\mathrm{T}_{\mathrm{x}} \mathrm{T}_{\mathrm{y}} \mathrm{T}_{\mathrm{z}} \mathrm{R}_{\mathrm{y}}\right)$, as sketched in Figure 1. A preliminary alignment of all parts was performed in air prior to the experiment, using the synchrotron visible light for autocollimation.

\subsection{Holographic Mask}

The preparation of the holographic mask relied on an original process developed at CSNSM [41] and used also for kirigami [42,43], which is illustrated in Figure 2. First, 
the object and reference apertures are opened in the direction perpendicular to the Aucoated and X-ray opaque $\mathrm{Si}_{3} \mathrm{~N}_{4}$ membrane by FIB milling (Figure 2a). Then an area of $\sim 50 \times 50 \mu \mathrm{m}^{2}$ containing the apertures is delimited and three of its sides are sliced by FIB (Figure $2 b$ ). Finally, the sliced area containing the holography apertures is lifted to the desired angle, at $45^{\circ}$ in our case (Figure 2c). Figure 2d shows that several of such masks, lifted at either $+45^{\circ}$ or $-45^{\circ}$, can be prepared on a single $\mathrm{Si}_{3} \mathrm{~N}_{4}$ multi-membrane Si substrate. Finally, Figure 2e shows an alternative mask design, comprising a line-reference aperture (30 $\mathrm{nm}$ wide in its narrowest part and $8 \mu \mathrm{m}$ long) for HERALDO data processing [44-46], an approach that was used already at the SEXTANTS beamline in transmission mode [47-49]. Data reported below make use of this mask.
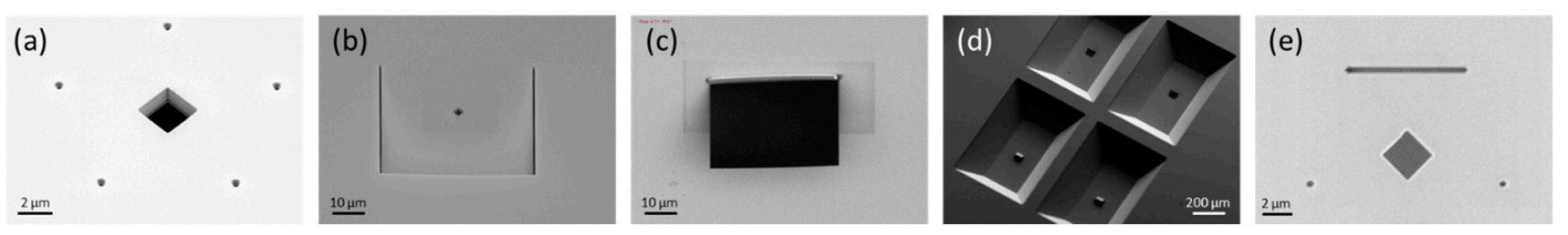

Figure 2. Scanning electron microscopy (SEM) images of the holographic mask fabrication process by focused ion beam milling. (a) Normal incidence etching of the holographic mask in the gold coated $\mathrm{Si}_{3} \mathrm{~N}_{4}$ membrane, with a square object aperture and five point-reference apertures. (b) Three-side cutting of a $50 \times 50 \mu^{2}$ area containing the holography apertures (view at $45^{\circ}$ from the surface). (c) The cut area containing the apertures is lifted at $45^{\circ}$ with respect to the membrane surface. (d) View of the four-membrane Si substrate, showing two masks lifted at $+45^{\circ}$ and two others at $-45^{\circ}$. (e) Mask design alternative to (a), comprising a $2.4 \mu \mathrm{m}$ square object aperture, plus two point-reference and one line-reference apertures.

A precise parallel positioning of the sample and mask surfaces (Figure 1) makes it possible to bring them in close contact, as in the transmission case $[18,24,26,27]$, gaining stability and avoiding (or at least reducing to a minimum) the need for back-propagation corrections [34].

For the top two masks shown in Figure 2d, X-rays go through the lifted mask first, impinge at $45^{\circ}$ on the sample positioned behind the mask, and are scattered towards the CCD detector placed at $90^{\circ}$. For the lower masks of Figure $2 \mathrm{~d}, \mathrm{X}$ rays enter the $50 \mu \mathrm{m}$ opening first, impinge on the sample at $45^{\circ}$, and the scattered beam goes through the holographic mask before reaching the detector. Data shown in Figure 3 were collected using this configuration, which also corresponds to the sketch of Figure 1.
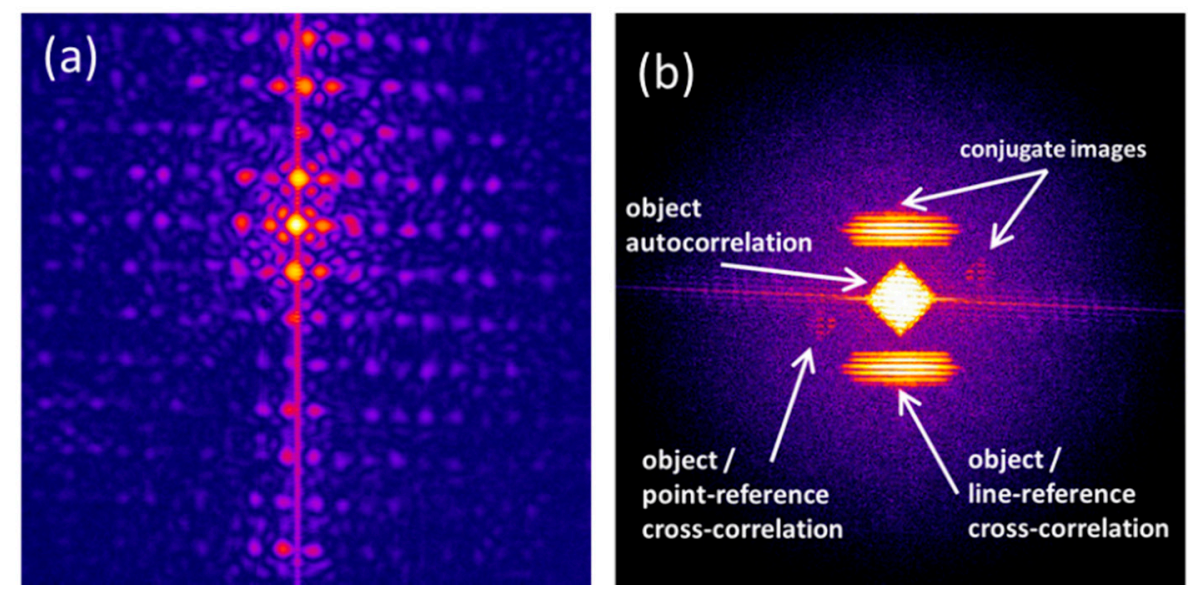

Figure 3. X-ray holography in reflection mode. (a) Hologram collected at $776 \mathrm{eV}$ and $90^{\circ}$ scattering angle from an array of sub- $\mu \mathrm{m} \mathrm{Co} / \mathrm{Cu}$ dots, using the holographic mask shown in Figure 2e, which comprises both line- and point-references. (b) Fourier transform of (a). The object autocorrelation is at the center of the image. The object/point-reference and object/line-reference cross-correlations are indicated by arrows in the lower half of the picture. Conjugate images appear in the upper half. 


\subsection{Ptychographic Mask}

The preparation of the ptychographic masks followed the standard procedure of FIB milling of the same $X$-ray opaque membranes. Several apertures, either square $(1.7 \mu \mathrm{m}$ and $2.4 \mu \mathrm{m}$ ) or circular (from 0.6 to $3 \mu \mathrm{m}$ in diameter) were prepared on the same multimembrane $\mathrm{Si}$ support. Only one aperture was illuminated at a time, selected by positioning it behind the $20 \mu \mathrm{m}$ guard-hole (Figure 1). By an appropriate shaping of the Si support, each mask could be aligned normal to the incident beam and positioned as close as $1.5 \mathrm{~mm}$ along the $X$ direction from the point where the photons impinge on the sample surface. The sample position behind the mask was scanned in sub- $\mu \mathrm{m}(100$ to $400 \mathrm{~nm})$ steps along both $Y$ and $Z^{\prime}$ directions within the sample surface (see Figure 1), using encoded piezoelectric motors. In this way several overlapping sample areas were illuminated and, for each sample position, the 2D map of the scattered intensity was collected by the CCD detector. One complete acquisition typically consisted of 40 to 120 steps. The entire data set was then treated by means of a phase retrieval algorithm that allows the reconstruction of the sample image over the whole probed area [50].

\section{Results}

\subsection{Holography}

Figure 3a shows the 2D diffraction pattern of the soft $\mathrm{X}$-ray beam being scattered by the $\mathrm{Co} / \mathrm{Cu}$ sample $\left(700 \times 300 \mathrm{~nm}^{2}\right.$ rectangles on a $880 \times 540 \mathrm{~nm}^{2}$ mesh $)$ and going through the holographic mask.

The photon energy is tuned to $776 \mathrm{eV}$, corresponding to the Co $2 \mathrm{p}_{3 / 2}$ resonance. In this reciprocal space image, one can clearly identify contributions from the array of rectangles (horizontal/vertical intensity spots), from the $45^{\circ}$ tilted square holographic object aperture (intensity along $\pm 45^{\circ}$ axes), and from the horizontal line-reference aperture (intensity scattered along the vertical line through the center of the figure). One can also observe fine modulations of the intensity corresponding to interference between these different contributions. Figure $3 \mathrm{~b}$ shows the Fourier transform of the scattered intensity providing a real space image, with the autocorrelation of the object aperture dominating its central part. The holographic mask design produces cross-correlations between object and reference apertures that are well separated from the autocorrelation. The cross-correlations between the object and point-reference apertures and between the object and line-reference apertures are indicated by arrows in Figure $3 b$. They are accompanied by conjugate images, symmetric with respect to the center of the figure [17]. Although Figure 3 suggests that we have appropriate conditions for achieving soft X-ray imaging by FTH in reflection mode, the high scattering angle implies that only a very low intensity goes through the holographic mask, especially through the point-reference aperture.

Figure 4 shows the details of the FT reconstructions obtained at $776 \mathrm{eV}$ using either the point-reference (Figure $4 \mathrm{~b}$ ) or the line-reference (Figure $4 \mathrm{c}, \mathrm{d}$ ), and compares the results with an image of the array of $\mathrm{Co} / \mathrm{Cu}$ rectangles obtained by scanning electron microscopy (SEM, Figure 4a).

In all the holographic images the objects can be identified, as highlighted also by the line profiles shown in Figure 4e,f. Due to the low signal to noise ratio (SNR), however, we can hardly claim to have obtained a clear image of the objects. 

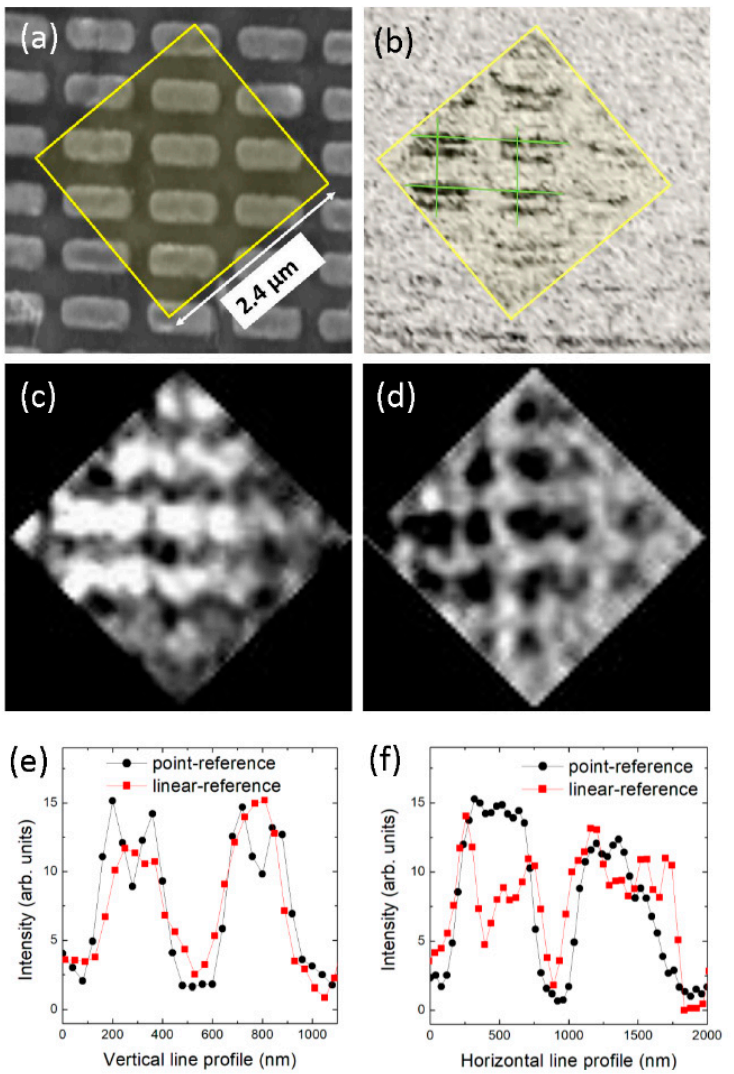

Figure 4. Reflection holography with point- and line-references. (a) SEM image of the Co/Cu patterned sample. The yellow square highlights an area equivalent to the object aperture of the holographic mask. (b) Cross-correlation image with the point-reference, where one can identify the sub-micron structures. (c,d) Images obtained by the HERALDO analysis $[44,45]$ of the object/linereference cross correlation. Two images are generated, with inverse contrast. (e,f) Comparison of vertical (e) and horizontal (f) line profiles obtained from images $(\mathbf{b}, \mathbf{c})$.

\subsection{Ptychography}

Concerning ptychography, the function of the mask is to define the X-ray source that illuminates a certain sample area. Several diffraction patterns are collected by moving with high precision the sample with respect to the mask, illuminating overlapping areas. Finally, an iterative algorithm for phase retrieval [50] treats the ensemble of the diffraction patterns to reconstruct a real space image of the amplitude and phase contrast corresponding to the probed area. Fairly good results have been obtained already at the SEXTANTS beamline using this approach in transmission mode [49].

A first set of data was recorded with the detector positioned at $90^{\circ}$ with respect to the incoming beam, measuring the scattering from a pattern of triangular holes in a permalloy film (Figure 5a), using a photon energy of $707 \mathrm{eV}$ (Fe $2 p$ resonance). The mask had a $2 \mu \mathrm{m}$ diameter aperture and was set normal to the incident beam, $1.5 \mathrm{~mm}$ away from the sample. Figure $5 \mathrm{~b}$ shows the amplitude image obtained, after 1300 iterations, from the analysis of 40 diffraction patterns. Next, the CCD was placed at $30^{\circ}$ with respect to the incoming beam, with a gain in scattered intensity that allowed us to speed up the acquisition and to increase the number of steps in the ptychography measurement. Figure $5 \mathrm{c}$ shows the image of the reconstructed amplitude (60 diffraction patterns, 1241 iterations) for the array of triangles. In spite of the gain in intensity, we obtained an image of worse quality at more grazing incidence, the reasons for that becoming apparent in the analysis of the data below. 

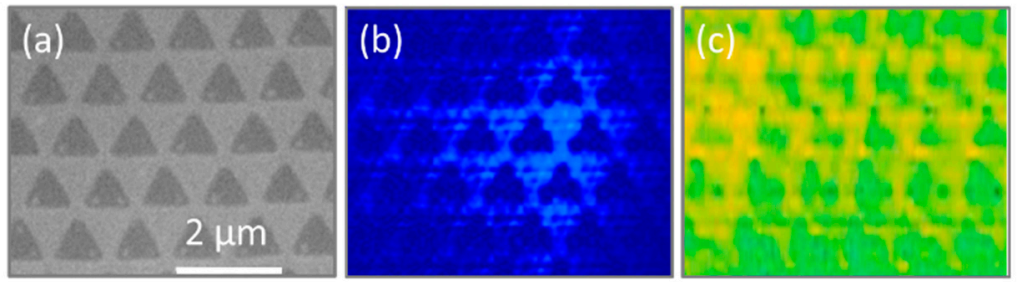

Figure 5. (a) SEM image of the permalloy pattern. Amplitude contrast images of the sample reconstructed from diffraction patterns acquired by ptychography at a grazing incidence $\omega=45^{\circ}$ (b) and $\omega=15^{\circ}$ (c). Reconstructions based on 40 and 60 diffraction patterns in (b,c), respectively. The photon energy was $707 \mathrm{eV}$ (Fe-2p resonance).

Figure 6a shows the amplitude image of FeRh $400 \mathrm{~nm}$ squares, based on the analysis of 10 frames measured in reflection ptychography at grazing incidence $\left(\omega=15^{\circ}\right)$ and at $707 \mathrm{eV}$ photon energy. The total acquisition time was $\sim 15^{\prime}$, and the reconstruction relied on 5000 iterations. Figure $6 \mathrm{~b}$ shows the horizontal profile ( $\mathrm{Y}$ direction) of the intensity. The analysis of the intensity steps corresponding to the edges of the FeRh objects shows that they extend over $110 \pm 10 \mathrm{~nm}$, setting an upper limit to the spatial resolution in the $\mathrm{Y}$ direction. Along the $\mathrm{Z}^{\prime}$ direction, corresponding to the intersection between the incidence plane and the sample surface, the effective pixel size is enlarged by a factor $\sim 3.2$ due to the reduced $Q_{Z^{\prime}}$ range covered by the detector for $\omega=15^{\circ}$ grazing incidence of the $X$ rays. As a result, it is not possible to discern any intensity plateau associated with the $400 \mathrm{~nm}$ objects in Figure $6 \mathrm{c}$. Therefore, the intensity gained by setting the detector at $30^{\circ}$ is counterbalanced by a loss of spatial resolution in the vertical direction with an overall degradation of the image quality, as shown also in Figure 5.

(a)

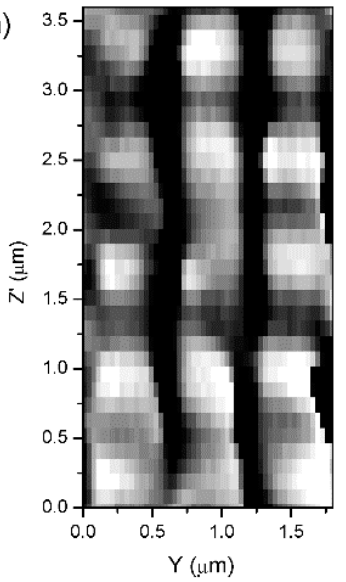

(b)

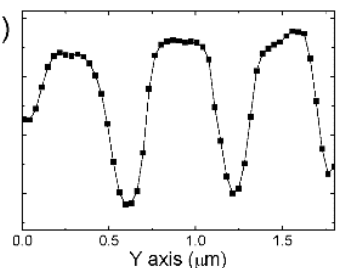

(c)

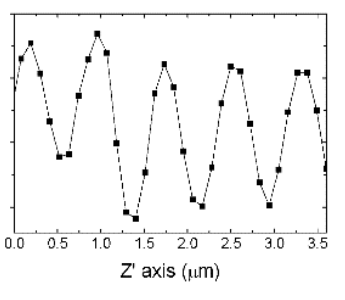

Figure 6. (a) Amplitude contrast image of the $\sim 400 \mathrm{~nm}$ FeRh squares on a $600 \times 800 \mathrm{~nm}^{2}$ mesh, reconstructed from 10 diffraction patterns acquired by ptychography at a grazing incidence $\omega=15^{\circ}$ and at a photon energy of $707 \mathrm{eV}$. The size of the reconstructed pixel is $34.4 \times 109.1 \mathrm{~nm}^{2}$. Horizontal (b) and vertical (c) line profiles are also shown.

\section{Discussion}

We consider that the reflection holography and ptychography experiments reported here prove the technical feasibility of this method, and that the results achieved are positive and encouraging. Although the quality of the images obtained so far is, admittedly, rather poor, they represent nonetheless a first achievement in the process of developing a new experimental approach.

Beyond providing a reasonable proof of feasibility, this experiment also allowed us to introduce a new and original approach to the mask fabrication process. Concerning holography, the new mask design represents a major step forward with respect to attempts made in the past. By moving the sample laterally, different sample areas can be brought 
into the field of view, and this approach makes it possible to extend the capabilities of the separate mask/sample FTH technique, already developed in transmission [18,24,27], to the reflection mode. Although the images we obtained so far are of rather modest quality, these results are extremely encouraging for continuing the development of reflection X-ray holography.

Ptychography poses less severe constraints than holography in terms of signal-tonoise ratio. These first attempts in reflection mode have yielded already better results, although not yet completely satisfactory in terms of image contrast and spatial resolution. In the case of the FeRh sample, the ion etching process may affect the X-ray reflectivity near the edges of the etched regions, with a negative influence on the image reconstruction of Figure 6. We cannot really quantify this effect for our measurements and, anyway, a similar observation would not apply to the permalloy sample of Figure 5.

Overall, the results achieved allow us to identify in the signal intensity the main limiting factor that has to be addressed in future reflection soft X-ray imaging experiments. This is particularly true for holography: even at an optimized synchrotron source, the intensity of the reference soft X-ray wave scattered at high angle through a $\sim 20-40 \mathrm{~nm}$ aperture requires extremely long exposure times, exacerbating noise and stability problems. Working at lower photon energy, e.g., at the 3 p resonances of $3 \mathrm{~d}-\mathrm{TM}$ (around $40-70 \mathrm{eV}$ ), is an interesting option for overcoming the reflected intensity problem and for achieving a better SNR. We could not explore this option in the present experiment because the fixed sample-detector distance of $470 \mathrm{~mm}$ implies covering a much-reduced Q-space at lower photon energy, producing very large pixels $(\sim 300 \mathrm{~nm})$ in the FTH images. A different setup, with the CCD set closer to the sample (50-100 mm), will be required for exploring the possibility of working in reflectivity at the $3 p$ resonances. Another approach, already tested in transmission [18,51], consists in the implementation of multiple reference apertures forming independent images that can be summed up to improve the SNR ratio.

We conclude by pointing out that all the ongoing upgrade projects at synchrotron sources have as a clear and declared objective the optimization of the coherent flux, therefore providing much improved conditions for lensless imaging techniques. Also, the high intensity per pulse offered by the X-ray FEL sources will represent a major asset for a drastic improvement of the SNR and for taking a decisive step forward in the development of soft X-ray imaging in reflection mode.

Author Contributions: Conceptualization, H.P., F.F., C.S., M.S.; methodology, H.P., F.F., R.D., C.S.; software, H.P.; investigation, all authors; data curation, H.P., M.S.; writing—original draft preparation, M.S.; writing — review and editing, all authors. All authors have read and agreed to the published version of the manuscript.

Funding: This research received no external funding.

Data Availability Statement: The data that support the findings of this study are available from the corresponding author upon reasonable request.

Acknowledgments: We acknowledge SOLEIL for provision of synchrotron radiation facilities. We thank K. Medjoubi (Synchrotron SOLEIL) for assistance in installing the phase retrieval computer code. We acknowledge the use of the SEM-FIB facility of the Institut des Sciences Moléculaires d'Orsay for mask preparation.

Conflicts of Interest: The authors declare no conflict of interest.

\section{References}

1. Fink, J.; Schierle, E.; Weschke, E.; Geck, J. Resonant elastic soft X-ray scattering. Rep. Prog. Phys. 2013, 76, 056502. [CrossRef]

2. Spezzani, C.; Ferrari, E.; Allaria, E.; Vidal, F.; Ciavardini, A.; Delaunay, R.; Capotondi, F.; Pedersoli, E.; Coreno, M.; Svetina, C.; et al. Magnetization and microstructure dynamics in Fe/MnAs/GaAs(001): Fe magnetization reversal by a femtosecond laser pulse. Phys. Rev. Lett. 2014, 113, 247202. [CrossRef]

3. Buzzi, M.; Först, M.; Mankowsky, R.; Cavalleri, A. Probing dynamics in quantum materials with femtosecond X-rays. Nat. Rev. Mater. 2018, 3, 299. [CrossRef] 
4. Holldack, K.; Pontius, N.; Schierle, E.; Kachel, T.; Soltwisch, V.; Mitzner, R.; Quast, T.; Springholz, G.; Weschke, E. Ultrafast dynamics of antiferromagnetic order studied by femtosecond resonant soft X-ray diffraction. Appl. Phys. Lett. 2010, 97, 062502. [CrossRef]

5. $\quad$ Pontius, N.; Kachel, T.; Schüßler-Langeheine, C.; Schlotter, W.F.; Beye, M.; Sorgenfrei, F.; Chang, C.-F.; Föhlisch, A.; Wurth, W.; Metcalf, P.; et al. Time-resolved resonant soft x-ray diffraction with free-electron lasers: Femtosecond dynamics across the Verwey transition in magnetite. Appl. Phys. Lett. 2011, 98, 182504. [CrossRef]

6. $\quad$ Ehrke, H.; Tobey, R.I.; Wall, S.; Cavill, S.A.; Först, M.; Khanna, V.; Garl, T.; Stojanovic, N.; Prabhakaran, D.; Boothroyd, A.T.; et al. Photoinduced melting of antiferromagnetic order in $\mathrm{La}_{0.5} \mathrm{Sr}_{1.5} \mathrm{MnO}_{4}$ measured using ultrafast resonant soft $\mathrm{X}$-ray diffraction. Phys. Rev. Lett. 2011, 106, 217401. [CrossRef]

7. Johnson, S.L.; De Souza, R.A.; Staub, U.; Beaud, P.; Möhr-Vorobeva, E.; Ingold, G.; Caviezel, A.; Scagnoli, V.; Schlotter, W.F.; Turner, J.J.; et al. Femtosecond dynamics of the collinear-to-spiral antiferromagnetic phase transition in CuO. Phys. Rev. Lett. 2012, 108, 037203. [CrossRef] [PubMed]

8. Kubacka, T.; Johnson, J.A.; Hoffmann, M.C.; Vicario, C.; de Jong, S.; Beaud, P.; Grübel, S.; Huang, S.-W.; Huber, L.; Patthey, L.; et al. Large-amplitude spin dynamics driven by a THz pulse in resonance with an electromagnon. Science 2014, 343, 1333-1336. [CrossRef] [PubMed]

9. Chauleau, J.-Y.; Legrand, W.; Reyren, N.; Maccariello, D.; Collin, S.; Popescu, H.; Bouzehouane, K.; Cros, V.; Jaouen, N.; Fert, A. Chirality in magnetic multilayers probed by the symmetry and the amplitude of dichroism in X-ray resonant magnetic scattering. Phys. Rev. Lett. 2018, 120, 037202. [CrossRef] [PubMed]

10. Chauleau, J.-Y.; Chirac, T.; Fusil, S.; Garcia, V.; Akhtar, W.; Tranchida, J.; Thibaudeau, P.; Gross, I.; Blouzon, C.; Finco, A.; et al. Electric and antiferromagnetic chiral textures at multiferroic domain walls. Nat. Mater. 2020, 19, 386-390. [CrossRef]

11. Lee, W.; Chuang, Y.; Moore, R.; Zhu, Y.; Patthey, L.; Trigo, M.; Lu, D.; Kirchmann, P.; Krupin, O.; Yi, M.; et al. Phase fluctuations and the absence of topological defects in a photo-excited charge-ordered nickelate. Nat. Commun. 2012, 3, 838. [CrossRef]

12. Chuang, Y.D.; Lee, W.S.; Kung, Y.F.; Sorini, A.P.; Moritz, B.; Moore, R.G.; Patthey, L.; Trigo, M.; Lu, D.H.; Kirchmann, P.S.; et al. Real-time manifestation of strongly coupled spin and charge order parameters in stripe-ordered $\mathrm{La}_{1.75} \mathrm{Sr}_{0.25} \mathrm{NiO}_{4}$ nickelate crystals using time-resolved resonant X-ray diffraction. Phys. Rev. Lett. 2013, 110, 127404. [CrossRef]

13. Mitrano, M.; Lee, S.; Husain, A.A.; Delacretaz, L.; Zhu, M.; Munoz, G.D.L.P.; Sun, S.X.-L.; Joe, Y.I.; Reid, A.H.; Wandel, S.F.; et al. Ultrafast time-resolved X-ray scattering reveals diffusive charge order dynamics in $\mathrm{La}_{2-x} \mathrm{Ba}_{x} \mathrm{CuO}_{4}$. Sci. Adv. 2019, 5. [CrossRef]

14. Ehrke, H.; Tobey, R.I.; Wall, S.; Cavill, S.A.; Prabhakaran, D.; Boothroyd, A.T.; Gensch, M.; Reutler, P.; Revcolevschi, A.; Dhesi, S.S.; et al. Ultrafast resonant soft X-ray scattering in manganites: Direct measurement of time-dependent orbital order. In Proceedings of the International Conference on Ultrafast Phenomena, Snowmass, CO, USA, 18-23 July 2010.

15. Zwiebler, M.; Borrero, J.E.H.; Vafaee, M.; Komissinskiy, P.; Macke, S.; Sutarto, R.; He, F.; Büchner, B.; Sawatzky, G.A.; Alff, L.; et al. Electronic depth profiles with atomic layer resolution from resonant soft x-ray reflectivity. New J. Phys. 2015, 17, 83046. [CrossRef]

16. Jal, E.; López-Flores, V.; Pontius, N.; Ferté, T.; Bergeard, N.; Boeglin, C.; Vodungbo, B.; Lüning, J.; Jaouen, N. Structural dynamics during laser-induced ultrafast demagnetization. Phys. Rev. B 2017, 95, 184422. [CrossRef]

17. Eisebitt, S.; Lüning, J.; Schlotter, W.F.; Lörgen, M.; Hellwig, O.; Eberhardt, W.; Stöhr, J. Lensless imaging of magnetic nanostructures by X-ray spectro-holography. Nat. Cell Biol. 2004, 432, 885-888. [CrossRef]

18. Spezzani, C.; Fortuna, F.; Delaunay, R.; Popescu, H.; Sacchi, M. X-ray holographic imaging of magnetic order in patterned Co/Pd multilayers. Phys. Rev. B 2013, 88, 224420. [CrossRef]

19. Beckers, M.; Senkbeil, T.; Gorniak, T.; Reese, M.; Giewekemeyer, K.; Gleber, S.-C.; Salditt, T.; Rosenhahn, A. Chemical contrast in soft X-ray ptychography. Phys. Rev. Lett. 2011, 107, 208101. [CrossRef] [PubMed]

20. Shapiro, D.A.; Yu, Y.-S.; Tyliszczak, T.; Cabana, J.; Celestre, R.; Chao, W.; Kaznatcheev, K.; Kilcoyne, A.; Maia, F.R.N.C.; Marchesini, S.; et al. Chemical composition mapping with nanometre resolution by soft X-ray microscopy. Nat. Photonics 2014, 8 , 765-769. [CrossRef]

21. Shi, X.; Fischer, P.; Neu, V.; Elefant, D.; Lee, J.C.T.; Shapiro, D.A.; Farmand, M.; Tyliszczak, T.; Shiu, H.-W.; Marchesini, S.; et al. Soft X-ray ptychography studies of nanoscale magnetic and structural correlations in thin $\mathrm{SmCo}_{5}$ films. Appl. Phys. Lett. 2016, 108, 094103. [CrossRef]

22. Vedmedenko, E.Y.; Kawakami, R.K.; Sheka, D.D.; Gambardella, P.; Kirilyuk, A.; Hirohata, A.; Binek, C.; Chubykalo-Fesenko, O.; Sanvito, S.; Kirby, B.J.; et al. The 2020 magnetism roadmap. J. Phys. D Appl. Phys. 2020, 53, 453001. [CrossRef]

23. Sacchi, M.; Spezzani, C.; Carpentiero, A.; Prasciolu, M.; Delaunay, R.; Luning, J.; Polack, F. Experimental setup for lensless imaging via soft $X$-ray resonant scattering. Rev. Sci. Instrum. 2007, 78, 43702. [CrossRef] [PubMed]

24. Stickler, D.; Frömter, R.; Stillrich, H.; Menk, C.; Tieg, C.; Streit-Nierobisch, S.; Sprung, M.; Gutt, C.; Stadler, L.-M.; Leupold, O.; et al. Soft X-ray holographic microscopy. Appl. Phys. Lett. 2010, 96, 042501. [CrossRef]

25. Tieg, C.; Frömter, R.; Stickler, D.; Hankemeier, S.; Philippi-Kobs, A.; Streit-Nierobisch, S.; Gutt, C.; Grübel, G.; Oepen, H.P. Imaging the in-plane magnetization in a Co microstructure by Fourier transform holography. Opt. Express 2010, 18, 27251-27256. [CrossRef]

26. Popescu, H.; Fortuna, F.; Delaunay, R.; Spezzani, C.; Lopez-Flores, V.; Jaouen, N.; Sacchi, M. Four-state magnetic configuration in a tri-layer asymmetric ring. Appl. Phys. Lett. 2015, 107, 202404. [CrossRef]

27. Sacchi, M.; Popescu, H.; Jaouen, N.; Tortarolo, M.; Fortuna, F.; Delaunay, R.; Spezzani, C. Magnetic imaging by Fourier transform holography using linearly polarized X-rays. Opt. Express 2012, 20, 9769-9776. [CrossRef] [PubMed] 
28. Willems, F.; Schmising, C.V.K.; Weder, D.; Günther, C.M.; Schneider, M.; Pfau, B.; Meise, S.; Guehrs, E.; Geilhufe, J.; Merhe, A.E.D.; et al. Multi-color imaging of magnetic Co/Pt heterostructures. Struct. Dyn. 2017, 4, 014301. [CrossRef] [PubMed]

29. Wang, T.; Zhu, D.; Wu, B.; Graves, C.; Schaffert, S.; Rander, T.; Müller, L.; Vodungbo, B.; Baumier, C.; Bernstein, D.P.; et al. Femtosecond single-shot imaging of nanoscale ferromagnetic order in $\mathrm{Co} / \mathrm{Pd}$ multilayers using resonant $\mathrm{X}$-ray holography. Phys. Rev. Lett. 2012, 108, 267403. [CrossRef]

30. Bergeard, N.; Schaffert, S.; Lopez-Flores, V.; Jaouen, N.; Geilhufe, J.; Günther, C.M.; Schneider, M.; Graves, C.; Wang, T.; Wu, B.; et al. Irreversible transformation of ferromagnetic ordered stripe domains in single-shot infrared-pump/resonant-x-ray-scatteringprobe experiments. Phys. Rev. B 2015, 91, 054416. [CrossRef]

31. Gardner, D.F.; Zhang, B.; Seaberg, M.D.; Martin, L.S.; Adams, D.E.; Salmassi, F.; Gullikson, E.; Kapteyn, H.; Murnane, M. High numerical aperture reflection mode coherent diffraction microscopy using off-axis apertured illumination. Opt. Express 2012, 20, 19050-19059. [CrossRef]

32. Zürch, M.; Kern, C.; Spielmann, C. XUV coherent diffraction imaging in reflection geometry with low numerical aperture. Opt. Express 2013, 21, 21131-21147. [CrossRef] [PubMed]

33. Roy, S.; Parks, D.; Seu, K.A.; Su, R.; Turner, J.J.; Chao, W.; Anderson, E.H.; Cabrini, S.; Kevan, S. Lensless X-ray imaging in reflection geometry. Nat. Photonics 2011, 5, 243-245. [CrossRef]

34. Guehrs, E.; Günther, C.M.; Pfau, B.; Rander, T.; Schaffert, S.; Schlotter, W.F.; Eisebitt, S. Wavefield back-propagation in highresolution X-ray holography with a movable field of view. Opt. Express 2010, 18, 18922-18931. [CrossRef]

35. Gerchberg, R.W.; Saxton, W.O. A practical algorithm for the determination of phase from image and diffraction plane pictures. Optik 1972, 35, 227.

36. Maiden, A.M.; Rodenburg, J. An improved ptychographical phase retrieval algorithm for diffractive imaging. Ultramicroscopy 2009, 109, 1256-1262. [CrossRef]

37. Pfeiffer, F. X-ray ptychography. Nat. Photonics 2017, 12, 9-17. [CrossRef]

38. Sacchi, M.; Popescu, H.; Gaudemer, R.; Jaouen, N.; Avila, A.; Delaunay, R.; Fortuna, F.; Maier, U.; Spezzani, C. IRMA-2 at SOLEIL: A set-up for magnetic and coherent scattering of polarized soft X-rays. J. Phys. Conf. Ser. 2013, 425, 202009. [CrossRef]

39. Sacchi, M.; Jaouen, N.; Popescu, H.; Gaudemer, R.; Tonnerre, J.M.; Chiuzbăian, S.G.; Hague, C.F.; Delmotte, A.; Dubuisson, J.M.; Cauchon, G.; et al. The SEXTANTS beamline at SOLEIL: A new facility for elastic, inelastic and coherent scattering of soft X-rays. J. Phys. Conf. Ser. 2013, 425, 072018. [CrossRef]

40. Spezzani, C.; Fabrizioli, M.; Candeloro, P.; Di Fabrizio, E.; Panaccione, G.; Sacchi, M. Magnetic order in a submicron patterned permalloy film studied by resonant X-ray scattering. Phys. Rev. B 2004, 69, 224412. [CrossRef]

41. Fortuna, F.; Spezzani, C.; Delaunay, R.; Popescu, H.; Sacchi, M. Testing a New Set-Up for X-ray Holographic Imaging in Reflectivity Mode. 2013. Available online: https://www.researchgate.net/publication/356361282_Testing_a_new_set-up_for_xray_holographic_imaging_in_reflectivity_mode?channel=doi\&linkId=6196ce2661f0987720b27f7e\&showFulltext=true (accessed on 10 December 2021). [CrossRef]

42. Li, J.; Liu, Z. Focused-ion-beam-based nano-kirigami: From art to photonics. Nanophotonics 2018, 7, 1637-1650. [CrossRef]

43. Chen, S.; Chen, J.; Zhang, X.; Li, Z.-Y.; Li, J. Kirigami/origami: Unfolding the new regime of advanced 3D microfabrication/nanofabrication with "folding". Light. Sci. Appl. 2020, 9, 75. [CrossRef]

44. Guizar-Sicairos, M.; Fienup, J. Holography with extended reference by autocorrelation linear differential operation. Opt. Express 2007, 15, 17592-17612. [CrossRef]

45. Zhu, D.; Guizar-Sicairos, M.; Wu, B.; Scherz, A.; Acremann, Y.; Tyliszczak, T.; Fischer, P.; Friedenberger, N.; Ollefs, K.J.; Farle, M.; et al. High-resolution X-ray lensless imaging by differential holographic encoding. Phys. Rev. Lett. 2010, 105, 043901. [CrossRef]

46. Duckworth, T.A.; Ogrin, F.; Dhesi, S.S.; Langridge, S.; Whiteside, A.; Moore, T.; Beutier, G.; Van Der Laan, G. Magnetic imaging by X-ray holography using extended references. Opt. Express 2011, 19, 16223-16228. [CrossRef]

47. Bukin, N.; McKeever, C.; Burgos-Parra, E.; Keatley, P.S.; Hicken, R.J.; Ogrin, F.Y.; Beutier, G.; Dupraz, M.; Popescu, H.; Jaouen, N.; et al. Time-resolved imaging of magnetic vortex dynamics using holography with extended reference autocorrelation by linear differential operator. Sci. Rep. 2016, 6, 36307. [CrossRef] [PubMed]

48. Loudon, J.C.; Twitchett-Harrison, A.C.; Cortés-Ortuño, D.; Birch, M.T.; Turnbull, L.A.; Štefančič, A.; Ogrin, F.Y.; Burgos-Parra, E.O.; Bukin, N.; Laurenson, A.; et al. Do images of biskyrmions show type-II bubbles? Adv. Mater. 2019, 31, e1806598. [CrossRef]

49. Popescu, H.; Perron, J.; Pilette, B.; Vacheresse, R.; Pinty, V.; Gaudemer, R.; Sacchi, M.; Delaunay, R.; Fortuna, F.; Medjoubi, K.; et al. COMET: A new end-station at SOLEIL for coherent magnetic scattering in transmission. J. Synchrotron Radiat. 2019, 26, 280-290. [CrossRef]

50. Medjoubi, K.; Baranton, G.; Somogyi, A. Fast full-field micro-tomography at the Nanoscopium multitechnique nanoprobe beamline of Synchrotron Soleil. Microsc. Microanal. 2018, 24, 254-255. [CrossRef]

51. Schlotter, W.F.; Rick, R.; Chen, K.; Scherz, A.; Stohr, J.; Luning, J.; Eisebitt, S.; Gunther, C.; Eberhardt, W.; Hellwig, O.; et al. Multiple reference Fourier transform holography with soft X-rays. Appl. Phys. Lett. 2006, 89, 163112. [CrossRef] 